\title{
Rational Reasoning or Adaptive Behavior? Evidence from Two-Person Beauty Contest Games*
}

\author{
Brit Grosskopf \\ Rosemarie Nagel \\ Texas A\&M University Universitat Pompeu Fabra
}

June 2007

\begin{abstract}
Many experiments have shown that human subjects do not necessarily behave in line with game theoretic assumptions and solution concepts. The reasons for this non-conformity are multiple. In this paper we study the argument whether a deviation from game theory is because subjects are rational, but doubt that others are rational as well, compared to the argument that subjects, in general, are boundedly rational themselves. To distinguish these two hypotheses, we study behavior in repeated 2-person and many-person BeautyContest-Games which are strategically different from one another. We analyze four different treatments and observe that convergence toward equilibrium is driven by learning through the information about the other player's choice and adaptation rather than self-initiated rational reasoning.
\end{abstract}

Journal of Economic Literature Classification Numbers: C7, C9

* Nagel acknowledges financial support from the Spanish Ministry of Education and Science under grants SEC2002-03403 and SEJ2005-08391, the Human Frontier Science Program HFCP RGP0056/2005-C and thanks the Barcelona Economics Program of CREA for support. Contact: Brit Grosskopf, Texas A\&M University, 4228 TAMU, College Station, TX 77843-4228, Email: bgrosskopf@econmail.tamu.edu. 


\section{Introduction}

Nash equilibrium is the most prevalent solution concept in game theory. However, many experiments have shown that human subjects do not necessarily behave in accordance with standard equilibrium predictions. There are many possible reasons for this. For example, a fully rational player might find the equilibrium, however he might doubt that all players will choose equilibrium strategies. This doubt can potentially be driven by two considerations, (a) the doubt about other players' rationality and (b) the belief that other players doubt the rationality of their co-players. ${ }^{1}$ Or, alternatively, players can be boundedly rational, maybe because of computational limits and therefore do not realize what the equilibrium behavior is. These two interpretations can be hard to separate observationally, since equilibrium strategies are not in general best replies to non-equilibrium choices of other players.

In this paper, we report an experiment that allows us to observationally distinguish between these two hypotheses. We focus on dominance solvable "beauty contest" games that have been a fruitful source of experiments concerned with assessing the bounds of human rationality. ${ }^{2}$ The results of such experiments typically conclude that human subjects play as if they were considering no more than a few iterated steps of reasoning. However, the two hypotheses mentioned above are fully confounded on these data: a player who can only think three steps ahead is observationally indistinguishable from a fully rational player who thinks that his opponents only think two steps ahead.

In a Beauty-Contest game (BCG) $n$ participants simultaneously choose a number from the interval $[0,100]$. The winner (who receives a fixed prize) is the person whose number is closest to a given proportion, say two thirds,

\footnotetext{
${ }^{1}$ Howard Raiffa defines this as asymmetric prescriptive-descriptive behavior (e.g. Raiffa, 1982). In order to prescribe a behavior, one needs to describe the other side's behavior. However, very often the reasoning process stops there and is asymmetric in the sense that it does not take into account the description of one's own behavior by the other side.

${ }^{2}$ E.g., Bosch, Montalvo, Nagel and Satorra (2002), Güth, Kocher and Sutter (2002), Ho, Camerer and Weigelt (1998), Kocher and Sutter (2005), Nagel (1995), Nagel (1998), Stahl (1996) and Sutter (2005). See Weber (2001) for a study of bounds on human rationality in a different game.
} 
of the average of all chosen numbers. If there is a tie, the prize is split between those who tie. So far, BCGs have been conducted on groups from 3 to several thousands. In Grosskopf and Nagel (2007) we introduce the two-person BCG. ${ }^{3}$

When $n$ is large the theoretical solution that all choose zero can be found by an infinite process of iterated elimination of weakly dominated strategies. ${ }^{4}$ Thus a rational player who wants to play zero has to suppose that all others also find the solution zero. The experimental evidence is that most players do not choose zero. Therefore, zero does not always win and a choice of a positive number can be consistent with the following two interpretations: (1) A player realizes that zero is the equilibrium choice however doubts that all players choose zero, and (2) a player does not realize that zero is the equilibrium choice.

In line with the interpretation of a deviation from equilibrium by a rational player, Bosch et al. (2002) analyze the comments by newspaper readers who participated in a one shot $n>2$ BCG. They find that almost half of the subjects who submit comments argue that zero is the equilibrium but $80 \%$ of those do not choose it because of the expectation that some other players are not choosing zero. However, most of these choose numbers below 10. Similarly, Plott (1996) argues that apparently irrational outcomes of markets could be the result of rational people expecting others to behave irrationally.

The special feature of the two-person BCG is that zero is a weakly dominant strategy and thus is always the winning number for any choice of the other player. Therefore, the expectation of the irrationality of others, given as a potential explanation for the observed deviation from equilibrium of a rational player in the $n>2 \mathrm{BCG}$, does not apply to the $n=2$ case. However, as shown in Grosskopf and Nagel (2007), students as well as more sophisticated participants (economic researchers at conferences) choose numbers that are significantly greater than zero when first confronted with the two-person BCG.

\footnotetext{
${ }^{3}$ Since our first collection of data on the $n=2$ BCG, Costa-Gomes and Crawford (2006) also conducted two-person BCGs. Their two-person games are, however, quite different, i.e. they are asymmetric, with different $p$-values for different players in the pair and have continuous payoffs. Their games are dominance solvable in 3-52 iterations.

${ }^{4}$ See Brandenburger and Keisler (2000) for a theoretical analysis of the finitely iterated elimination of weakly dominated strategies and its requirements.
} 
The aim of the current paper is to investigate what it takes for participants to learn to choose zero in the two-person BCG. By manipulating the information feedback after each round we explore whether and how subjects converge to the optimal choice of zero and under which conditions this is the case. We are especially interested in whether subjects consciously learn to play the equilibrium or rather arrive at it through a process of adaptation. This analysis enables us to relate the observations to behavior in the $n>2$ case and derive potential reasons behind positive choices there.

We study four treatments of a $2-$ person $B C G$, in which fixed pairs repeatedly interact. The treatments differ by the feedback that players receive after each round, and whether the $n=2 \mathrm{BCG}$ is played before or after the $n>2$ BCG. The feedback is tailored to separate adaptation behavior that might be triggered by observing the other player's choice (e.g. through imitation) from pure outcome based adaptation (change one's choice in response to own payoff information only). The order in which the BCGs are played is manipulated in order to investigate learning transfer. Participants understanding the dominance argument can be separated from those who "learned" to choose zero without understanding it.

We observe that if players are given very detailed feedback about choices of their own opponent in the $n=2 \mathrm{BCG}$, the majority of them learn to play the weakly dominant strategy. This unraveling process is much more noisy if the amount of information is restricted to the own payoff. Choices do not unravel when no feedback is given.

Most players in the full information treatment increase their choices when switching from the $n=2$ BCG to the $n>2$ BCG. This is in line with an interpretation that players doubt the rationality of others in the $n>2$ case while having (finally) understood the dominance argument in the $n=2$ case. However, when the BCG's are played in the reverse order, no evidence to uphold this argument can be found. Players who switch from the $n>2 \mathrm{BCG}$ to the $n=2$ BCG also increase their choices, clearly violating dominance.

We conclude that players do not apply rational reasoning but are predominantly boundedly rational. In particular, they do not search for dominant or dominated strategies but rather start with focal point choices and their best reply. The observation that the overwhelming majority chooses dominated strategies in the 2 -person game implies the predominance of bounded 
rationality in the $n>2 \mathrm{BCG}$ rather than a consideration that others being boundedly rational.

The paper is organized as follows. Section 2 analyzes the game and its theoretical solution in more detail and section 3 describes the experimental design. Section 4 discusses the experimental results and section 5 concludes. An Appendix includes a sample set of instructions.

\section{The Game}

In this study we focus on the BCG where the winning number is defined as the number that is closest to two thirds of the mean of the chosen numbers. The game is repeated for several periods. The unique equilibrium in the one-shot and in the repeated $n=2 \mathrm{BCG}$ is that both players choose zero. A unilateral deviation leads to a lower payoff, since zero is always closer to $2 / 3$ of the average of zero and also to $2 / 3$ of the average if the opponent chooses any positive number. Hence, zero weakly dominates all positive numbers. Thus, a rational player should choose zero without the need to rely on the assumption of common knowledge of rationality.

This is the essential difference between the $n=2$ and the $n>2$ person BCG. In the $n>2$ BCG a rational player should delete all weakly dominated strategies (numbers greater $2 / 3 \cdot 100$ ). However, further deletion relies upon his knowledge or belief of the other players' rationality and their beliefs of other players, hence zero as the unique best response relies on the common knowledge of rationality.

\section{Experimental Design}

All experiments were conducted in the experimental laboratory (LEEX) of the Universitat Pompeu Fabra in Barcelona. Since the BCG is sometimes used as a teaching tool in undergraduate economics, we carefully selected participants and made sure they were not previously exposed to the BCG. We only allowed freshmen to participate and solicited students from different campuses for different sessions. The experiments were conducted during April, September, October 2000, October 2004 and June 2005 using first year undergraduate students majoring in either economics, political science, 
law, medicine or humanities with no formal training in game theory at the University Pompeu Fabra in Barcelona. ${ }^{5}$

In an experimental session we had either ten, sixteen or eighteen students. Participants were first randomly matched into pairs in three of the four treatments. The $n=2$ BCG was repeated 10 times in a fixed pair setting, followed by 4 rounds of the $n>2$ BCG. $^{6}$ In a fourth treatment we reversed the order, i.e. first four rounds of the $n>2$ BCG were played followed by 10 rounds of the $n=2$ BCG. $^{7}$ The treatments further differed according to the information (feedback after each round) that was provided. Table I summarizes the experimental design.

\begin{tabular}{cc}
\hline \hline & $\boldsymbol{n}=\mathbf{2} \Longrightarrow \boldsymbol{n}>\mathbf{2}$ \\
\hline Full Info & 2 sessions $(18+16)$ \\
Partial Info & 3 sessions $(2 \times 18+10)$ \\
No Info & 3 sessions $(2 \times 18+16)$ \\
\hline \hline & $\boldsymbol{n}>\mathbf{2} \Longrightarrow \boldsymbol{n = 2}$ \\
\hline Reverse Full Info & 2 sessions $(2 \times 18)$ \\
\hline
\end{tabular}

Table I: Summary of treatments

(number of participants given in parentheses)

Full Info Treatment: In the Full Info treatment all players were informed about the other player's choice in the pair $(n=2)$ or all other players' choices in the group $(n>2)$. The experimenter wrote those choices as well as $2 / 3$ of the mean and the obtained payoff either on the decision sheet in the case

\footnotetext{
${ }^{5}$ All sessions run in 2005 only used non econ students and were computerized using zTree (Fischbacher, 1999).

${ }^{6}$ The exact size of $n$ was equal to the respective session size, i.e. 10, 16 or 18 .

${ }^{7} \mathrm{~A}$ translated set of instructions is available in the Appendix. In the set of instructions for the computerized experiments we included "The winner will be determined as follows: First, the average number of both participants' choices (including yours) will be computed. This average will be computed by adding up the numbers chosen by both participants in a pair and dividing by two. A target number will then be determined by multiplying the average of a pair's choices by two-thirds. Thus: target number $=2 / 3 x$ (average of everyone's choices in a pair). The person who chose closest to this target number will be the winner. In case of a tie, the prize is split amongst the winners."
} 
of $n=2$ or on the board in front of the room in the case of $n>2$ after each round. ${ }^{8}$

Partial Info Treatment: After each round, participants in the Partial Info treatment were only informed whether they had won the fixed prize or not. ${ }^{9}$ Learning through the choice of the other was therefore excluded by design.

These first two treatments were chosen to separate adaptation behavior triggered by observing the other player's choice from adaptation behavior in response to an observed win or loss outcome. The third treatment was designed to investigate whether behavior changes only in response to outcomes or in response to the mere repetition of the task. We therefore omitted all feedback in the third treatment, similar to Weber (2003).

No Info Treatment: Participants did not receive any feedback in the No Info treatment. The experimenters recorded each participant's choice, calculated the average and target number and determined the winner. Participants were informed that the experimenters had done this (but were not told the results) and were then asked to make a choice for the next period. Only at the conclusion of both parts of the experiment were subjects informed whether they had obtained the fixed prize in any given period (or parts of it).

In order to better understand transfer behavior between the 2-person and many-person BCG and to compare first round behavior in the 2-person BCG with the many-person BCG when both are played first, we conducted a fourth treatment where we reversed the order of the BCG's.

\footnotetext{
${ }^{8}$ In the computerized session this information was provided on the individual computer screens.

${ }^{9}$ In case of a tie, winning subjects split the fixed prize and were informed about the amount they obtained.
} 
Reverse Full Info: In the Reverse Full Info, participants first played four rounds of the $n>2$ BCG followed by ten rounds of the $n=2$ BCG. We gave subjects the same information as in the Full Info treatment.

The fixed prize in each round was equal to 100 pesetas (at the time roughly $\$ 0.64$ ) in the $n=2$ BCG. ${ }^{10}$ We paid the winner of each round 1,000 pesetas in the $n>2$ BCG. In the later experiments, conducted after the introduction of the Euro, we paid a 1 Euro prize (at that time about $\$ 1.20$ ) in the $n=2$ BCG and 10 Euros in the $n>2$ BCG. Subjects were paid their accumulated earnings at the end of the experiment. Average earnings were about 1,250 pesetas (at that time about $\$ 8$ ), including a 500 peseta show-up fee. In the later sessions the average earnings were 11.20 Euros (at that time about \$13.44), including a 4 Euro show-up fee. Sessions lasted about 60 minutes with the computerized ones being shorter.

In all treatments we asked participants to explain their choice. ${ }^{11}$ While we know little how this might actually affect the choice itself, we hoped that such an effect would not interact with any potential treatment effects. We asked for explanations in order to gain some inside into the reasoning process.

\section{Experimental Results}

\subsection{First Round Behavior}

Figure 1 shows the cumulative frequencies of choices in the first round of all four treatments. We find that the distributions of first round choices in the three treatments where the $n=2 \mathrm{BCG}$ was played first are not significantly different from each other (Kruskal-Wallis test statistic: $\chi^{2}=3.546$, dof $=2$, $p=0.1698$ ). Only $9.85 \%$ (13 out of 132 ) of the participants choose zero in the first round. Thus, roughly $90 \%$ play dominated strategies, which is probably the highest amount ever observed in an experimental game. $21.21 \%$ (28 out of 132 ) of the chosen numbers are above 50.

\footnotetext{
${ }^{10}$ While the 100 pesetas may seem like a small amount in $\$$-equivalents it would buy a delicious café con leche (latté) in the cafeteria of Pompeu Fabra.

${ }^{11}$ Participants were asked to provide handwritten explanations in both, the hand-run as well as the computerized, sessions.
} 
Surprisingly, initial choices in the Reverse Full Info treatment - where participants first played 4 rounds of the $n>2$ BCG followed by 10 rounds of the $n=2 \mathrm{BCG}$ - are not stochastically different from those in the $n=2$ BCG (i.e. round 1, Reverse Full Info versus round 1, Full Info) (KolmogorovSmirnov test, $p=0.094)$.
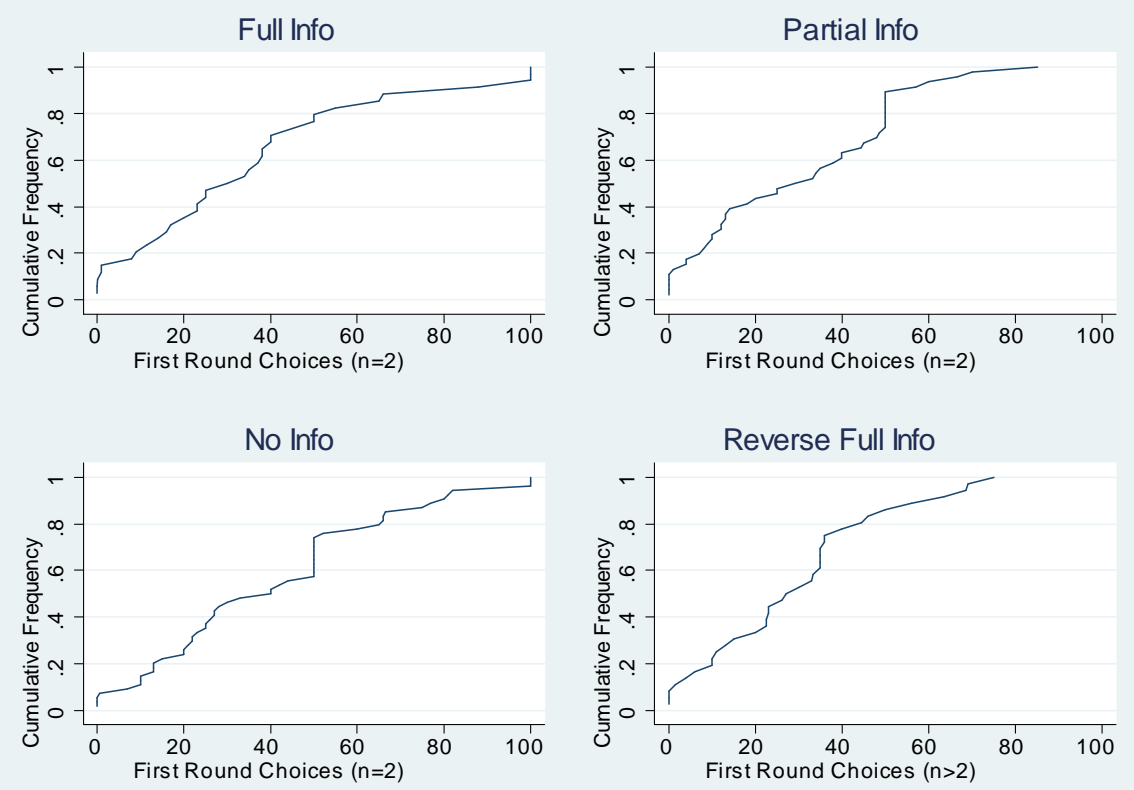

Figure 1: Cumulative Frequencies of First Round Choices

\subsection{Behavior over Time $(n=2)$}

Figure 2 plots the median choice over all pairs for each treatment over time. ${ }^{12}$ Figure 3 shows individual choices. In the Full Info treatment choices clearly unravel to the equilibrium (Spearman correlation coefficient for the aggregate data is $-0.98, p<0.001)$. $76.5 \%(13 / 17)$ of the pairs have a significant negative correlation coefficient. In round 10 we observe $73.5 \%(25 / 34)$ choices to be equal to zero.

\footnotetext{
${ }^{12}$ We have chosen the median instead of the mean since we believe it to be more descriptive of the distribution of choices.
} 


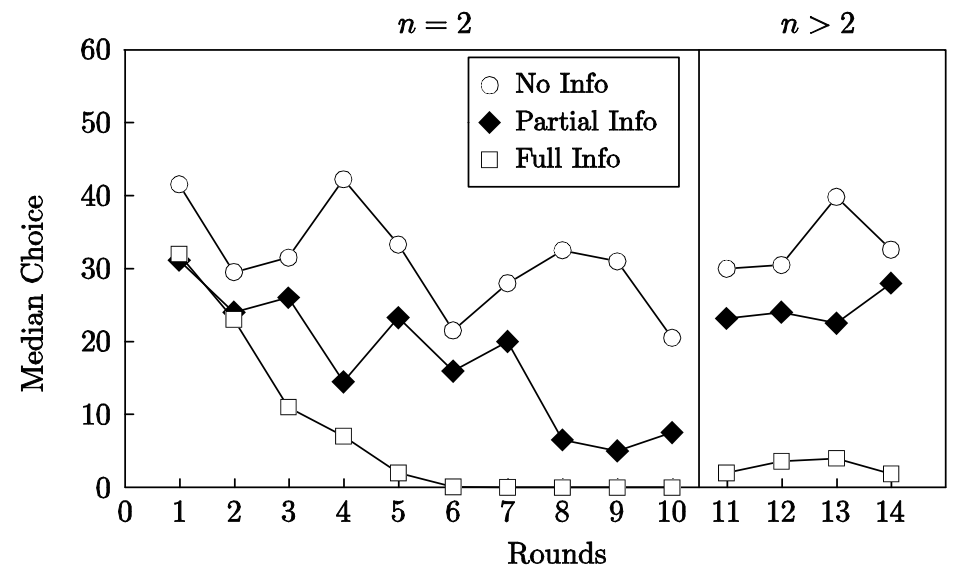

Figure 2: Median Choices over Time

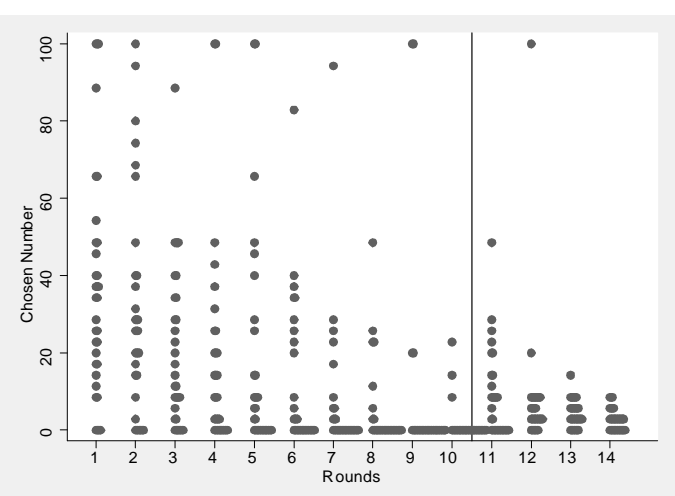

(A) Full Info

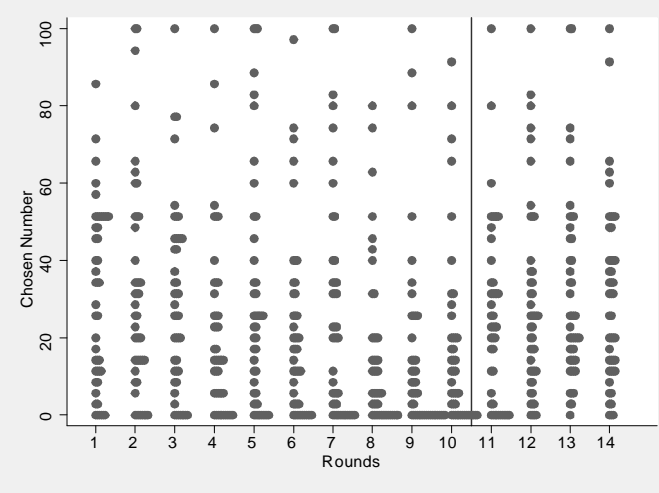

(B) Partial Info

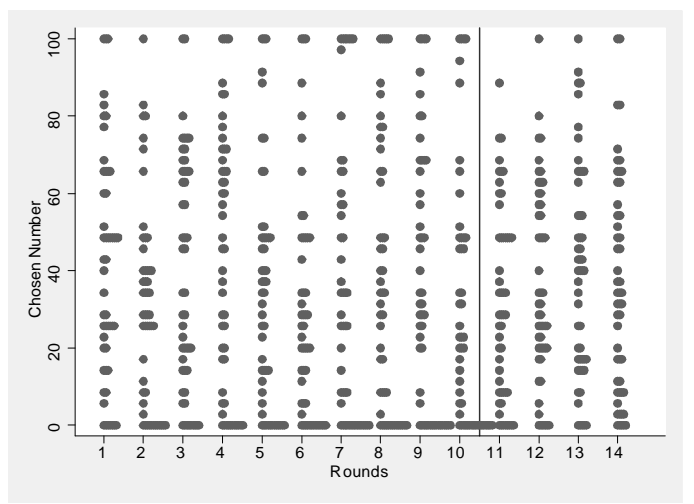

(C) No Info

Figure 3: Dotplots of Individual Choices

( $\mathrm{x}$-axes refer to rounds and $\mathrm{y}$-axes plot the distribution of choices) 
In the Partial Info treatment, the unraveling to equilibrium is much slower (Spearman correlation coefficient for the aggregate data is $-0.87, p<0.01$ ). Only $21.7 \%$ (5/23) of all pairs have a significantly negative coefficient. This proportion is significantly less than in the Full Info treatment (Test of equality of proportions, $z=3.34, p<0.05) .{ }^{13}$ In round 10 we observe $26.1 \%(12 / 46)$ choices to be equal to zero compared to $73.5 \%$ in the Full Info treatment. This difference in proportions is significant (Test of equality of proportions, $z=4.21, p<0.001)$.

Finally, there is no significant decrease to zero in the No Info treatment (Spearman correlation coefficient for the aggregate data is 0.0027, $p=0.9416) .{ }^{14}$ Only $7.7 \%(2 / 26)$ of all pairs have a significant negative coefficient, and $3.9 \%(1 / 26)$ are even significantly positive. However, there is only a marginal difference between the Partial and No Info treatments (Test of equality of proportions, Partial vs. No Info: $z=1.47, p=0.0708$ ). In round 10 we observe $25.0 \%$ (13/52) choices to be equal to zero compared to $26.1 \%$ in the Partial Info treatment. These proportions are not significantly different from one another (Test of equality of proportions, $z=0.12$, n.s.).

How can the low tendency to play the equilibrium and the rather slow convergence or non-convergence be explained? The analysis shown in Table II is an attempt to determine the driving forces behind the observed behavior. We analyze the sequential dependencies in the data by looking at individual behavior from one round to the next. We count the number of observations with increased, unchanged and decreased choices. We do this separately for winners and losers. For winners we distinguish between those who chose zero and those chose a number other than zero. Losers who decrease their choices are further divided into different categories depending on whether

\footnotetext{
${ }^{13}$ The specific test statistic is $z=\left(p_{1}-p_{2}\right) / S_{p_{c}}$, where $p_{i}$ is the proportion of pairs with a significant negative correlation coefficient in subsample $i$, and $S_{p_{c}}=$ $\sqrt{p_{c}\left(1-p_{c}\right)\left(\frac{1}{N_{1}}+\frac{1}{N_{2}}\right)}$ is an estimate of the standard error of the difference in proportions, $p_{1}-p_{2} . p_{c}$ is an estimate of the population proportion under the null hypothesis of equal proportions, $p_{c}=\left(p_{1} N_{1}+p_{2} N_{2}\right) /\left(N_{1}+N_{2}\right)$, where $N_{i}$ is the total number of pairs in subsample $i$ (see Glasnapp and Poggio, 1985).

${ }^{14}$ Note that this is different from the results obtained by Weber (2003). Weber (2003) finds unraveling in $n>2$ BCGs when no feedback is provided between rounds.
} 
the opponent's choice of the previous period was not quite reached, imitated, undercut with a choice above zero or whether zero was directly chosen.

\begin{tabular}{|c|c|c|c|}
\hline & Full Info ${ }^{15}$ & Partial Info ${ }^{16}$ & No $\operatorname{Info}^{17}$ \\
\hline \# Losers (L) & 124 & 196 & 232 \\
\hline$\# \mathrm{~L} \nearrow$ & $18.6 \%\left(\frac{23}{124}\right)$ & $39.3 \%\left(\frac{77}{196}\right)$ & $33.6 \%\left(\frac{78}{232}\right)$ \\
\hline$\# \mathrm{~L}=$ & $6.5 \%\left(\frac{8}{124}\right)$ & $7.1 \%\left(\frac{14}{196}\right)$ & $16.0 \%\left(\frac{37}{232}\right)$ \\
\hline$\# \mathrm{~L} \searrow^{*}$ & $75.0 \%\left(\frac{93}{124}\right)$ & $\mathbf{5 3 . 6 \%}\left(\frac{105}{196}\right)$ & $50.4 \%\left(\frac{117}{232}\right)$ \\
\hline$>$ other $^{a}$ & $38.7 \%\left(\frac{48}{124}\right)$ & $33.2 \%\left(\frac{65}{196}\right)$ & $34.9 \%\left(\frac{81}{232}\right)$ \\
\hline$=$ other $\& \neq 0$ & $0.8 \%\left(\frac{1}{124}\right)$ & $1.0 \%\left(\frac{2}{196}\right)$ & $0.4 \%\left(\frac{1}{232}\right)$ \\
\hline$=$ other $\&=0$ & $9.7 \%\left(\frac{12}{124}\right)$ & $2.0 \%\left(\frac{4}{196}\right)$ & $1.3 \%\left(\frac{3}{232}\right)$ \\
\hline$<$ other $\&>0$ & $21.0 \%\left(\frac{26}{124}\right)$ & $15.8 \%\left(\frac{31}{196}\right)$ & $12.1 \%\left(\frac{28}{232}\right)$ \\
\hline$<$ other $\&=0$ & $4.8 \%\left(\frac{6}{124}\right)$ & $1.5 \%\left(\frac{3}{196}\right)$ & $1.7 \%\left(\frac{4}{232}\right)$ \\
\hline \# Winners $(\mathbf{W})>0$ & 68 & 122 & 139 \\
\hline$\# \mathrm{~W} \nearrow^{* *}$ & $22.1 \%\left(\frac{15}{68}\right)$ & $45.9 \%\left(\frac{56}{122}\right)$ & $49.6 \%\left(\frac{69}{139}\right)$ \\
\hline$>$ other & $7.4 \%\left(\frac{5}{68}\right)$ & $12.3 \%\left(\frac{15}{122}\right)$ & $19.4 \%\left(\frac{27}{139}\right)$ \\
\hline$=$ other & $1.5 \%\left(\frac{1}{68}\right)$ & $1.6 \%\left(\frac{2}{122}\right)$ & $0.7 \%\left(\frac{1}{139}\right)$ \\
\hline$<$ other & $13.2 \%\left(\frac{9}{68}\right)$ & $32.0 \%\left(\frac{39}{122}\right)$ & $29.5 \%\left(\frac{41}{139}\right)$ \\
\hline$\# \mathrm{~W}=$ & $11.8 \%\left(\frac{8}{68}\right)$ & $18.9 \%\left(\frac{23}{122}\right)$ & $7.9 \%\left(\frac{11}{139}\right)$ \\
\hline$\#$ & $66.2 \%\left(\frac{45}{68}\right)$ & $35.3 \%\left(\frac{43}{122}\right)$ & $42.4 \%\left(\frac{59}{139}\right)$ \\
\hline$>0$ & $47.1 \%\left(\frac{32}{68}\right)$ & $30.3 \%\left(\frac{37}{122}\right)$ & $33.1 \%\left(\frac{46}{139}\right)$ \\
\hline$=0$ & $19.1 \%\left(\frac{13}{68}\right)$ & $4.9 \%\left(\frac{6}{122}\right)$ & $9.4 \%\left(\frac{13}{139}\right)$ \\
\hline \# Winners $(\mathbf{W})=0$ & 56 & 74 & 93 \\
\hline$\# \mathrm{~W} \nearrow^{* *}$ & $10.7 \%\left(\frac{6}{56}\right)$ & $5.4 \%\left(\frac{4}{74}\right)$ & $15.1 \%\left(\frac{14}{93}\right)$ \\
\hline$>$ other & $7.1 \%\left(\frac{4}{56}\right)$ & $2.7 \%\left(\frac{2}{74}\right)$ & $5.4 \%\left(\frac{5}{93}\right)$ \\
\hline$=$ other & $0 \%\left(\frac{0}{56}\right)$ & $0 \%\left(\frac{0}{74}\right)$ & $0 \%\left(\frac{0}{93}\right)$ \\
\hline$<$ other & $3.6 \%\left(\frac{2}{56}\right)$ & $2.7 \%\left(\frac{2}{74}\right)$ & $9.7 \%\left(\frac{9}{93}\right)$ \\
\hline$\# \mathrm{~W}=$ & $89.3 \%\left(\frac{50}{56}\right)$ & $94.6 \%\left(\frac{70}{74}\right)$ & $84.9 \%\left(\frac{79}{93}\right)$ \\
\hline
\end{tabular}

Table II: Sequential Dependencies

\footnotetext{
${ }^{15}$ Out of 153 pairs analyzed, $29(18.95 \%)$ tied, with 26 ties at zero $(89.66 \%)$.

${ }^{16}$ Out of 207 pairs analyzed, $11(5.31 \%)$ tied, with 7 ties at zero $(63.64 \%)$.

${ }^{17}$ Out of 236 pairs analyzed, $2(0.85 \%)$ tied, with one tie at zero $(50 \%)$ and one at 50 .
} 
We do a similar analysis for winners who increase their choices. For winners who won with a number other than zero we also distinguish between those who decrease their choice to a number above zero and those who choose zero. The modal frequencies within each group in a treatment are in bold. The percentages of losers and winners choosing zero (i.e., playing the equilibrium) after a choice above zero are emphasized.

The highest frequency of change is observed when losers receive full information: $75 \%$ decrease their choices. Fewer losers in the other two treatments decrease their choices (53.6\% in Partial Info and 50.4\% in No Info). Note that while participants in the No Info treatment did not know whether they lost or won, we included this information for comparability with the other treatments. Losers in the Partial Info, therefore, do not seem to react to the information of a loss since their behavior is similar to the behavior of "losers" in the No Info treatment.

Learning to play the equilibrium, however, is difficult even in the Full Info treatment. Only $14.6 \%$ (out of the $75 \%$ ) of the losers who lower their choice decrease it to zero $(9.7 \%$, who imitate the winner, plus $4.8 \%$, who undercut the winner). The corresponding percentages in the other two treatments are $3.5 \%$ out of $53.6 \%$ (Partial Info) and $3 \%$ out of $50.4 \%$ (No Info). More than $1 / 3$ of the losers do not lower their choices below the choice of the winning partner in all treatments.

Choosing zero seems equally difficult for winners who have not chosen zero. Only $19.1 \%$ of the $66.2 \%$ decreasing winners chose zero in the next round in the Full Info treatment. In the other two treatments the corresponding frequencies are much lower, $4.9 \%$ and $9.4 \%$. However, once winners reach the equilibrium, they choose it again with high probability in all treatments $(89.3 \%, 94.6 \%$, and $84.9 \%$ in Full Info, Partial Info and No Info respectively, see last row of Table II).

Imitation of the winning number is fairly low in the Full Info treatment $(0.8 \%+9.7 \%=10.5 \%)$ as compared to the frequency of players understanding that a number below the number of the winner should be chosen $(21 \%$ $+4.8 \%=25.8 \%$ ). However, it is obviously much higher than in the Partial Info treatment $(3 \%)$ and the No Info treatment $(1.7 \%)$.

Players who imitate a winning choice greater than zero could be interpreted as "naïve" players. Losers who undercut the winner but do not yet 
choose zero could be thought of as level 1 players (see Nagel, 1995; Stahl, 1996 and Ho et al., 1998 for a classification of players according to their levels of reasoning). Players who are playing zero by undercutting winners are very high level players or so-called equilibrium players. Those who stay unchanged or even increase have no understanding of the game.

Another evidence that players who undercut the winner with a choice of zero are more "sophisticated" (i.e., exhibit higher levels of reasoning) can be found in the observation that all of the 6 observations in the Full Info treatment come from 6 players who chose zero in all of the subsequent rounds. The 12 observations of imitating choice of zero come from 8 individuals, 5 of which chose zero always after and 3 kept going up and down, again sometimes imitating the winner.

Taking all these observations together, we see that the majority of losers and winners decrease their choices in the Full Info treatment. This is not the case in the Partial and No Info treatments. While roughly half of the losers do decrease their choices, winners predominantly increase their choices in those treatments. There is also a big difference across treatments as to by how much decreasing losers and winners lower their choices. A combined $22.5 \%(31 / 138)$ of the decreasing losers and winners in the Full Info treatment choose zero. This happens less in the Partial Info $(8.8 \%, 13 / 148)$ and the No Info $(11.4 \%, 20 / 176)$ treatments. The additional information in the Full Info treatments allows for imitative behavior but also seems to induce higher levels of reasoning.

However, since it takes 7 rounds for the median choice to be zero in the Full Info treatment (the only treatment where we find clear convergence) we conclude that choices unravel to equilibrium through an adaptive learning process that is triggered by observing the other player's choice rather than by rational reasoning. Observed behavior of two sample pairs (shown in Figure 4) supports and illustrates this claim. Panel (A) shows a pair that slowly and parallel adjusts to equilibrium. In round 9 both happened to choose 1 and tied. In round 10, player 1 writes, "I choose 1 again because the other also did this last time and we tied. He will do the same again." However, player 2's argument in round 10 is "Since the other is doing what I do, we will both choose zero now." Player 4 in panel (B) of Figure 4 understands the game in round 7 and writes "It is always the lower number 
that wins and zero is the lowest possible." However, his counterpart does not understand the situation fully. He writes in round 10 "Well, since the other always won with zero, I want to see whether I also win by choosing zero."

Figure 5 shows behavior of two sample pairs in the Partial Info treatment. Lacking information about the other player's choice, participants' adaptation towards equilibrium is impeded. In panel (A) player 1 understood the game by round 2, player 2's comment about his round 10 choice is that "I choose 17 because it was a very special day for me on a personal level." Player 4 in panel (B) of Figure 5 won in round 9 by choosing 8 and writes in round 10 (where he also chooses 8) "I just do the same again, since I was successful in the last round." His counterpart chooses 5 in round 10 and says "I just try a low number."

Figure 6 gives a flavor of the "clueless" behavior in the No Info treatment. If participants have neither choice nor outcome feedback, no adjustment towards equilibrium occurs.

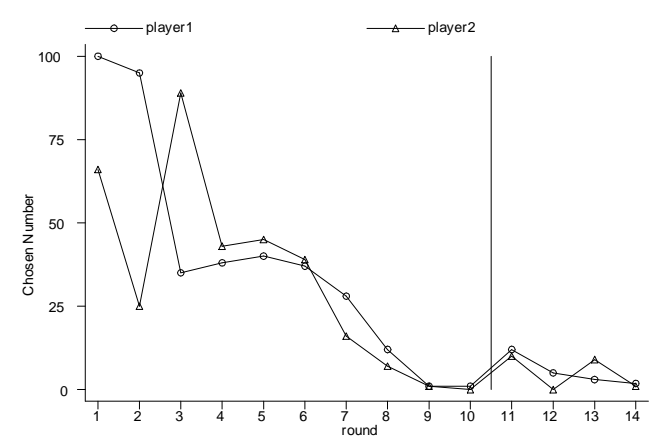

(A)

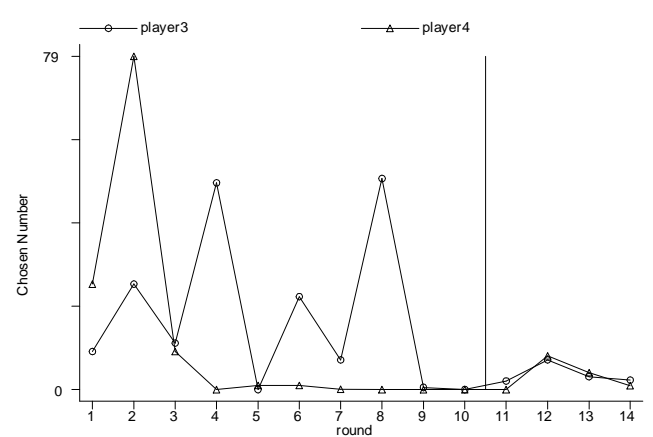

(B)

Figure 4: Choices of two sample pairs in the Full Info treatment 


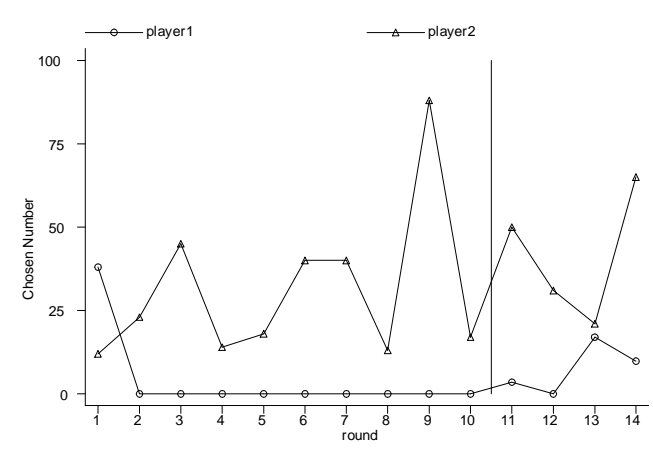

(A)

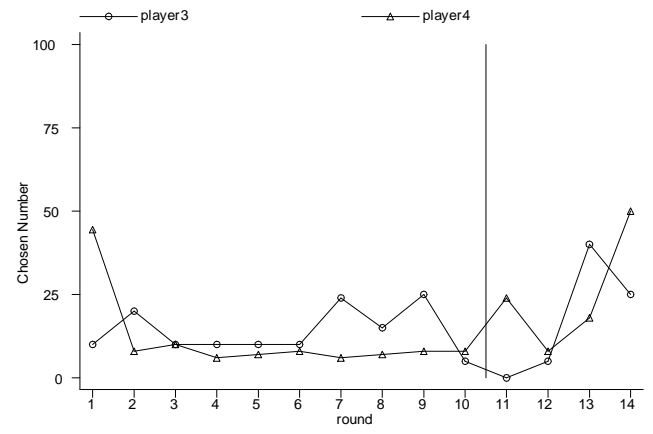

(B)

Figure 5: Choices of two sample pairs in the Partial Info treatment

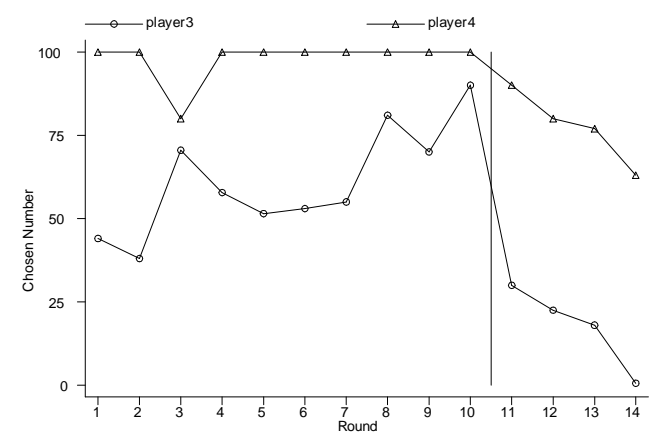

(A)

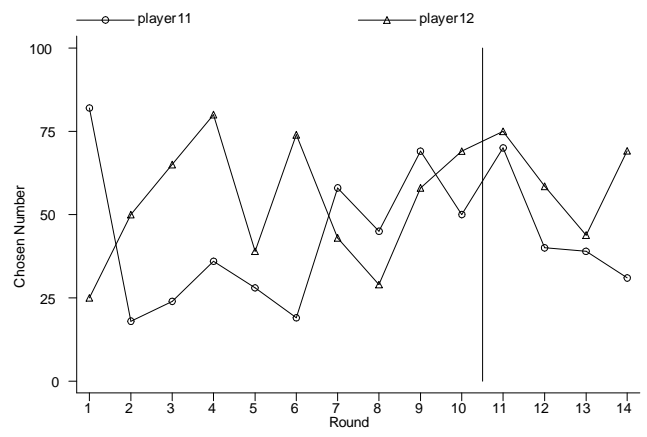

(B)

Figure 6: Choices of two sample pairs in the No Info treatment

\subsection{Behavior over Time $(n>2)$}

In all treatments where the $n=2 \mathrm{BCG}$ is played before the $n>2 \mathrm{BCG}$ we observe no significant decrease over time in the $n>2$ BCG (see Figure 2). This is unusual compared to behavior normally observed in other BCG games. ${ }^{18}$ Players seem to think that they are starting too low after having played the $n=2$ BCG and adjust their choices upwards. We speculate that this initial "overreaction" would go away over time. We would expect to see choices unravel if the $n>2$ BCG had been played longer.

\footnotetext{
${ }^{18}$ Spearman correlation coefficients are negative in the Full Info, and positive in the Partial and No Info treatments. However, none are significantly different from zero.
} 


\subsection{Transfer Behavior: Change from $n=2$ to $n>2$}

In the Full and Partial Info treatments choices increase significantly when players switch from playing the $n=2$ BCG to the $n>2$ BCG (Sign test of means of matched pairs, Full Info: $p=0.0001$ and Partial Info: $p=0.0392$ ). However, choices do not differ in the two BCGs in the No Info treatment (Sign test of means of matched pairs, No Info: $p=0.4225)$. This suggests that while players in both information treatments realize that there is a difference between the two versions of the BCG, players in the No Info treatment treat them as behaviorally identically.

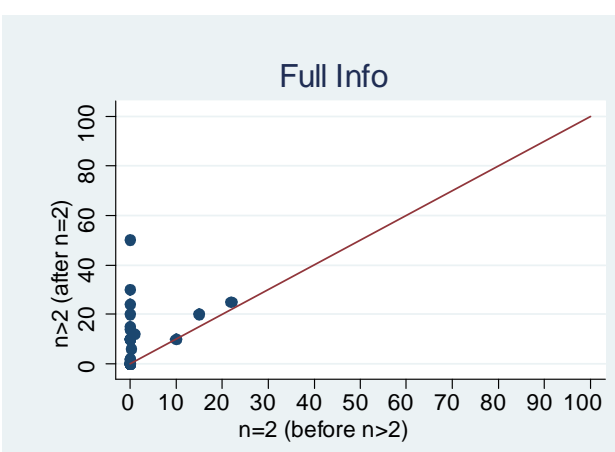

No Info

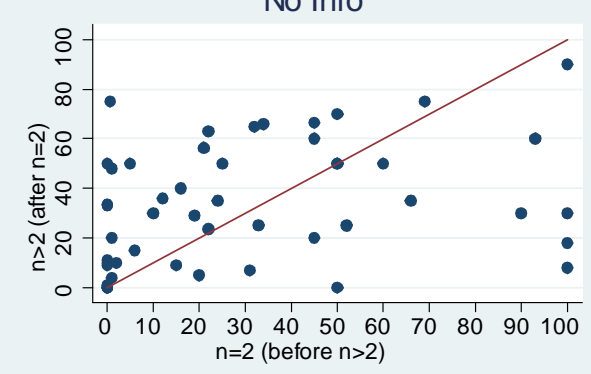

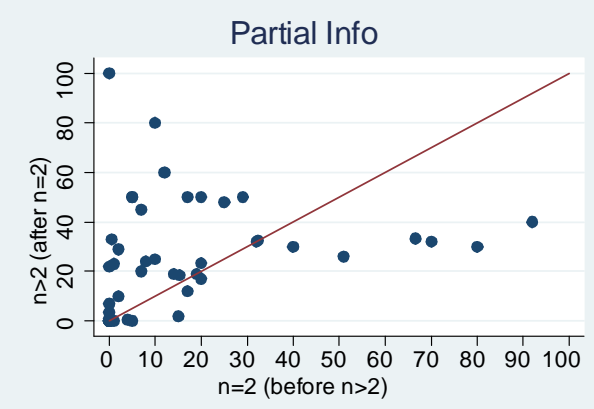

Reverse Full Info

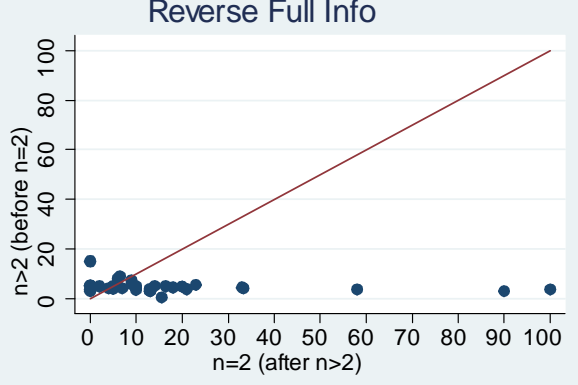

Figure 7: Transition Plots:

Period 10 to $11(n=2 \rightarrow n>2)$ and Period 4 to $5(n>2 \rightarrow n=2)$

(x-axes plot choices in $n=2$ and $\mathrm{y}$-axes plot choices in $n>2$ )

We further find that the distribution of choices in the Partial Info treatment in round 11 is significantly different from the distribution of choices in the corresponding round of the Full Info treatment (Kolmogorov-Smirnov test: $p=0.005)$. In fact, the median in the Partial Info treatment is significantly higher than in the Full Info treatment (Robust rank order test, 
$\grave{U}=-4.37, p<0.01)$. Surprisingly, no difference is found between choices in the Partial Info and the No Info treatment (Kolmogorov-Smirnov test: $p=0.256){ }^{19}$

The above mentioned observations can be seen in three of the panels of Figure 7 (Full Info, Partial Info and No Info) where an individual's choice in the last round of $n=2$ is plotted against the same individual's choice in the first round of $n>2$. So far, the evidence seems to suggest that players can learn to differentiate between the different BCGs when given appropriate feedback. The question remains whether they really understand the dominance argument? To address that question, we analyze whether it matters which BCG is played first. If players understand the difference between the two BCGs and in particular dominance, we should see no order effects. However, looking at the fourth panel of Figure 7 (Reverse Full Info) reveals almost a mirror image of the behavior observed in the Full Info treatment suggesting a strong order effect which we shall now analyze further.

\subsection{Order Effects}

Figure 8 plots the median of choices in the Reverse Full Info treatment where participants first played 4 rounds of the $n>2$ BCG followed by 10 rounds of the $n=2$ BCG. Figure 9 illustrates the distribution of individual choices and demonstrates clear unraveling in both parts of this treatment (Spearman correlation coefficient is $-0.6473, p<0.0001$ for rounds $1-4$ and -0.5266 , $p<0.0001$ for rounds $5-14$ ).

\footnotetext{
${ }^{19}$ On an individual level we find that no choices are above 50 in round 11 in the Full Info treatment, while there were $6.52 \%$ (3 out of 46 ) such choices in the Partial Info treatment and even $21.15 \%$ (11 out of 52 ) in the No Info treatment.
} 


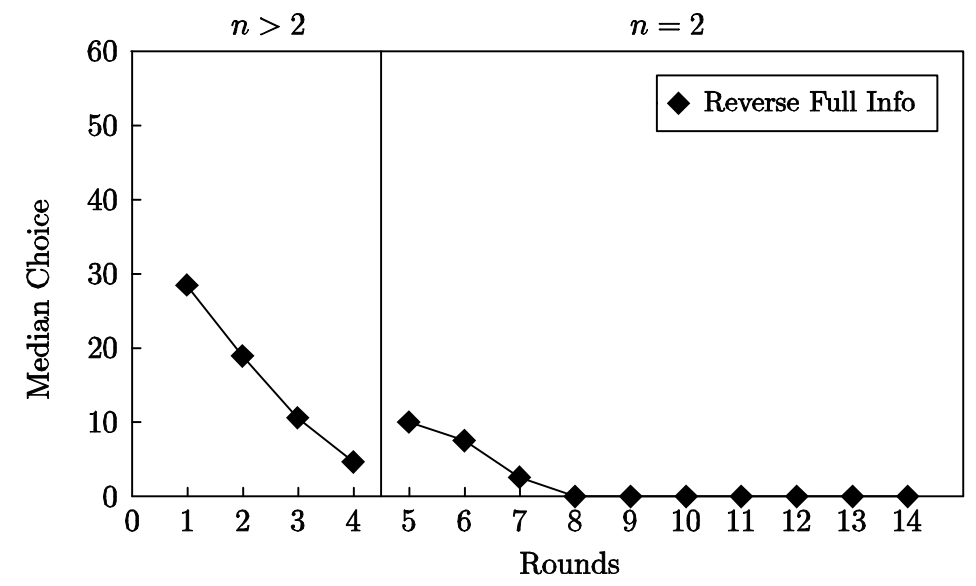

Figure 8: Median choices in the Reverse Full Info treatment $(n>2$ is played first followed by $n=2)$

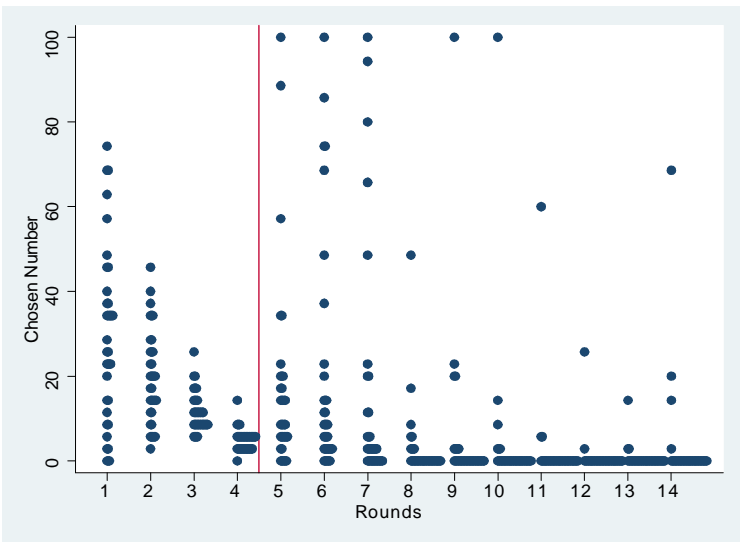

Figure 9: Dotplot of choices in the Reverse Full Info treatment

( $\mathrm{x}$-axes refer to rounds and $\mathrm{y}$-axes plot the distribution of choices)

While data from the Full Info treatment seem to suggest that most players understand dominance by round 10 (i.e. choose higher numbers in $n>2$ in round 11 than they do in the $n=2$ BCG in round 10), data from the Reverse Full Info treatment contradicts this because most players choose higher numbers in the $n=2$ BCG in round 5 than they do in the $n>2$ BCG in round 4 (see Figure 10; Sign test, $p=0.0083$ ). There is clearly a different kind of learning transfer going on in both treatments. 
In both treatments (Full Info as well as Reverse Full Info) choices are increased when the "new" BCG starts. The increase in the Full Info treatment can therefore not be attributed to the understanding of dominance and the mere doubt that others are rational. It looks more like a general "restart" effect that occurs independently of the order. ${ }^{20}$

Not surprisingly, comparing the first round of the $n>2$ BCG when it is played first (i.e., round 1, Reverse Full Info) with the first round of the $n>2$ BCG when it is played after the $n=2$ BCG (i.e. round 11, Full Info) we find that the distribution of means of pairs in the Full Info treatment is significantly lower than in the Reverse Full Info treatment (KolmogorovSmirnov test: $p<0.0001){ }^{21}$ Similarly, we find that choices in the $n=2$ BCG when it is played after the $n>2 \mathrm{BCG}$ are lower than when it is played without any prior experience (Kolmogorov-Smirnov test: $p=0.015$ ).

Another interesting observation from the Reverse Full Info treatment is that the unraveling in the $n=2 \mathrm{BCG}$ is quicker when it is played after the $n>2$ BCG. Here the median of choices is equal to zero starting from the fifth round, compared to round 8 in the Full Info treatment when $n=2$ BCG is played first. As mentioned before, we do not find any unraveling in the $n>2 \mathrm{BCG}$ when it is played after the $n=2 \mathrm{BCG}$ and clear unraveling when it is played before the $n=2 \mathrm{BCG}$.

\section{Discussion}

In all versions of the BCG that have been conducted (in this paper and elsewhere in the literature) we have seen that the majority of players do not choose the equilibrium zero in the very first period. However, players can "learn" to choose zero. The convergence in $n>2$ BCGs has been found to

\footnotetext{
${ }^{20} \mathrm{We}$ do, however, observe $16.7 \%$ (6/36) of the players in the Reverse Full Info treatment to change from a choice greater than zero in the $n>2$ BCG to zero in the $n=2$ BCG. Similarly, we observe $23.5 \%$ (8/34) of the players in the Full Info treatment to change from zero in the $n=2 \mathrm{BCG}$ to a choice greater than zero in the $n>2 \mathrm{BCG}$. This is consistent with being rational but doubting that others are rational.

${ }^{21}$ For independence reasons we can only use means of pairs in the case when the $n>2$ is played after the $n=2$ case. We therefore also calculate means of pairs in the Reverse Full Info treatment, although pairs have not yet been assigned. We use the pairing that is used in the $n=2$ segment of the Reverse Full Info treatment. Results do not change if we use all 17 sets of 9 independent pairs that are possible from each set of 18 observations.
} 
depend on the influence a single player has on the result, i.e. whether or not (s)he can "screw up" the outcome. ${ }^{22}$ Behavior in the median game (Duffy and Nagel, 1997) converges fastest to equilibrium. The small group (Ho et al., 1998) and the maximum game (Duffy and Nagel, 1997) have the slowest convergence.

In our 2-person BCG, a single player has the greatest influence on the result and can even win the game for sure. We therefore expect behavior to converge to the equilibrium. We observe this to be the case, however, the type of information feedback heavily influences the speed of convergence (see Full versus Partial Info). Without the appropriate feedback, very little convergence can be observed (see No Info).

Even though observed behavior in the Full Info treatment unravels quickly, this is mainly driven by the fact that at least one player in a pair finds the right solution that is then imitated. In case of the absence of a rational player, players typically adapt slowly, choosing lower and lower numbers. Our experimental results suggest that most players do not find the rational solution by rational reasoning but rather by adaptation. In this process a loser (somebody who has chosen the bigger number in the pair) easily understands that he has to decrease his choice. When information about the other player's choice is given, higher levels of reasoning are triggered and losers tend to lower their choices below the other's choice. However, when information about winning or losing is not given, a large number of players do not adapt to the game theoretic solution.

We conclude that the persistence of choices above zero in the $2-$ person game implies the predominance of bounded rationality in an $n$-person game rather than a consideration that others are boundedly rational. We have seen that the adaptation process is heavily influenced by the type of feedback that is given to participants after each round. Kenneth J. Arrow already pointed out that: "Information may be supplied socially, but to be used, it has to be absorbed individually. The limits on the ability to acquire information are a major barrier to it's diffusion. This line of argument leads to Herbert Simon's concept of bounded rationality as a process in time (p.8, 1994)."

\footnotetext{
${ }^{22}$ It also has been shown that groups converge faster than individuals, e.g. Kocher and Sutter (2005) and Sutter (2005).
} 


\section{REFERENCES}

1. Arrow, K. J. (1994): "Methodological Individualism and Social Knowledge," American Economics Review, 84, 1-9.

2. Bosch-Domenech, A., J. G. Montalvo, R. Nagel and A. Satorra (2002): "One, Two, (Three), Infinity, ...: Newspaper and Lab Beauty-Contest Games," American Economic Review, 92, 1687-1701.

3. Brandenburger, A. and H. J. Keisler (2001): "Epistemic Conditions for Iterated Admissibility," in Theoretical Aspects of Rationality and Knowledge: Proceedings of the Eighth Conference, edited by Johan van Benthem, Morgan Kaufmann, 31-37.

4. Costa-Gomes, M. and V. P. Crawford (2006): "Cognition and Behavior in Two-Person Guessing Games: An Experimental Study," American Economic Review, 96, 1737-1768.

5. Chen, Z. and M. W. Daehler (1998): "Positive and negative transfer in analogical problem solving by 6-year old children," Cognitive Development, 4, 327-344.

6. Duffy, J. and R. Nagel (1997): "On the Robustness of Behavior in Experimental Beauty-Contest Games," Economic Journal, 107, 16841700.

7. Fischbacher, U. (1999): "z-Tree: Zurich Toolbox for Ready-made Economic Experiments," Experimental Economics, forthcoming.

8. Glasnapp, D. and J. Poggio (1985): Essentials of Statistical Analysis for the Behavioral Sciences. Merrill, Columbus.

9. Grosskopf, B. and R. Nagel (2007): "The Two-Person Beauty Contest," Games and Economic Behavior, forthcoming.

10. Güth, W., M. Kocher and M. Sutter (2002): "Experimental 'Beauty Contests' with homogeneous and heterogeneous players and with interior and boundary equilibria," Economics Letters, 74, 219-228.

11. Ho, T., Camerer, C. and K. Weigelt (1998): "Iterated Dominance and Iterated Best Response in Experimental ' $p$-Beauty Contests'," American Economic Review, 88, 947-969. 
12. Kocher, M. and M. Sutter (2005): "The decision maker matters: Individual versus team behavior in experimental beauty-contest games," Economic Journal, 115, 200-223.

13. Luchins, A. S. and E. H. Luchins (1970): "Wertheimer's Seminars Revisited: Problem Solving and Thinking," Albany N.Y., S.U.N.Y.

14. Nagel, R. (1995): "Unraveling in Guessing Games: An Experimental Study," American Economic Review, 85, 1313-1326.

15. Plott, C. (1996): Rational Individual Behavior in Markets and Social Choice Processes: The Discovered Preference Hypothesis. In Arrow, K., Colombatto, E., Perlaman, M., and Schmidt, C., editors, The Rational Foundations of Economic Behavior. Macmillan: London.

16. Raiffa, H. (1982): The Art and Science of Negotiation. Harvard University Press.

17. Stahl, D. O. (1996): "Boundedly Rational Rule Learning in a Guessing Game," Games and Economic Behavior, 16, 303-330.

18. Sutter, M. (2005): "Are four heads better than two? An experimental beauty-contest game with teams of different size," Economics Letters, $88,41-46$.

19. Weber, R. A. (2001): "Behavior and learning in the 'dirty faces' game," Experimental Economics, 4(3), 229-242.

20. Weber, R. A. (2003): "Learning with no feedback in a competitive guessing game." Games and Economic Behavior, 44, 134-144. 


\section{Appendix - Instructions}

You are participating in an experiment on decision making. ${ }^{23}$ If you follow the instructions and apply them carefully, you can earn some money additionally to the 500 Pesetas [4 Euros] which we will give you in any case. From now on you are not allowed to talk to any participant in the experiment. If you have a question, please raise your hand and one of the instructors will attend you. Please, do not speak out aloud. Thank you.

\section{Rules $[n=2]$}

Today's experimental session consists of 2 parts. The first part will last for 10 rounds. At the beginning, we will randomly assign you into pairs of 2 participants. These assignments will stay constant over all 10 rounds of the first experiment, but none of you will know with whom you are paired. [Instructions for the second part were only distributed after the first part was finished.]

\section{Rules $[n>2]$}

This part consists of 4 rounds. All participants belong to the same group. The group is the same for all 4 rounds.

\section{Decisions $[n=2]$}

Each participant has to choose a number between 0 and 100 that can be decimal (including the extrema). The "winner" of each pair will be the person who selects a number that is closest to $2 / 3$ of the mean of the numbers chosen in that pair. [Additional sentences in all experimental sessions conducted in 2004 and 2005: The winner will be determined as follows: First, the average number of both participants' choices will be computed. This average will be computed by adding up the numbers chosen by both participants in a pair and dividing by two. A target number will then be determined by multiplying the average of a pair's choices by two-thirds.]

\footnotetext{
${ }^{23}$ Comments that were not necessarily in the instructions or parts of a different treatment are given in []. Original instructions were in Spanish.
} 


\section{Decisions $[n>2]$}

Each participant has to choose a number between 0 and 100 that can be decimal (including the extrema). The "winner" of the group will be the person who selects a number that is closest to $2 / 3$ of the mean of all numbers chosen in the group. [Additional sentences in all experimental sessions conducted in 2004 and 2005: The winner will be determined as follows: First, the average number of all participants' choices will be computed. This average will be computed by adding up the numbers chosen by all participants in the group and dividing by the number of people in the group. A target number will then be determined by multiplying the average of a pair's choices by two-thirds.]

\section{Payments in Points}

The winner of each pair receives 100 points. In case of a tie, the points are divided between the winners, that is each receives 50 points.

The winner of the entire group will receive 1000 points. In case of a tie, the points are split amongst those who tie.

\section{Payments in Pesetas [Euros]}

All points accumulated during the experiment will be converted into pesetas at the following exchange rate: 1 point is equal to 1 pta. [0.01 Euro], that is if you have accumulated 100 points, you will be paid 100 ptas [1 Euro].

\section{Information after each Round}

[Info] After each round we will inform each participant about the points (s)he has gained, the number chosen by the other person and $2 / 3$ of the mean [the other participants' choices in $n>2$ ].

[Partial Info] After each round we will inform each participant about the points (s)he has gained.

[No Info] There will be no information after each round. At the end of the second experiment of today's session, you will be informed about your earnings. 


\section{Sample Decision Sheets}

\section{Full Info Treatment}

\begin{tabular}{|c|c|c|c|c|c|}
\hline \multirow[b]{2}{*}{ Round } & & & \multicolumn{3}{|c|}{ To be filled in by the instructor } \\
\hline & $\begin{array}{l}\text { Number } \\
\text { chosen }\end{array}$ & $\begin{array}{c}\text { Why? } \\
\text { Please explain your reasoning! }\end{array}$ & $\begin{array}{l}\text { Number chosen } \\
\text { by the other }\end{array}$ & $2 / 3$ of the mean & Points \\
\hline 1 & & & & & \\
\hline$\vdots$ & & & & & \\
\hline 10 & & & & & \\
\hline & & & & Total & \\
\hline
\end{tabular}

\section{Partial Info Treatment}

\begin{tabular}{|c|c|c|c|}
\cline { 3 - 4 } Round & $\begin{array}{c}\text { Number } \\
\text { chosen }\end{array}$ & To be filled in by the instructor \\
\hline 1 & & & Why? \\
Please explain your reasoning! & Points \\
\hline$\vdots$ & & & \\
\hline 10 & & & Total: \\
\cline { 3 - 4 }
\end{tabular}

No Info Treatment

\begin{tabular}{|c|c|c|}
\hline Round & $\begin{array}{c}\text { Number } \\
\text { chosen }\end{array}$ & $\begin{array}{c}\text { Why? } \\
\text { Please explain your reasoning! }\end{array}$ \\
\hline 1 & & \\
\hline$\vdots$ & & \\
\hline 10 & & \\
\hline
\end{tabular}

